Aus der geburtshilfl. Klinik und dem medizinisch-chemischen Laboratorium der Universität $z u$ Tomsk.

\title{
Ueber die Kreatinin- und Kreatinausscheidung durch den Harn bei Wöchnerinnen.
}

\author{
Von
}

\section{Privatdozent Dr. J. Wakulenko.}

Kürzlich habe ich eine Reihe von Untersuchungen über die Ausscheidung des endogenen Kreatinins bei normalen Männern und Frauen ausgeführt, die in einem der nächsten Hefte der "Arbeiten des medizinisch-chemischen Laboratoriums der kaiserlichen Universität zu Tomsk" (russisch) veröffentlicht werden sollen.

Aus diesen Untersuchungen ergab sich unter anderem, dass Frauen sowohl absolut, als auch relativ weniger Kreatinin mit dem Harn ausscheiden, als Männer, natürlich kreatininfreie Nahrung vorausgesetzt.

Als Mittel aus meinen an acht Männern, die auf kreatininfreie Kost gesetzt waren, ausgeführten Bestimmungen fand ich die Tagesmenge des mit dem Harn ausgeschiedenen Kreatinins zu 1,41 g, was $0,022 \mathrm{~g}$ pro Tag and Kilo Körpergewicht ausmachte.

Diese Zahlen stehen in bestem Einklang mit den Werten, die in neuerer Zeit auch von anderer Seite angegeben sind. So fand Folin ${ }^{1}$ ) im Mittel aus seinen Untersuchungen an 13 Männern die Tagesausscheidung des Kreatinins mit dem Harn $=1,48 \mathrm{~g}$. Die Kreatininmenge pro Tag und Kilo ist bei ihm dieselbe, wie die von mir gefundene.

Closson²) bestimmte an sechs Männern pro Tag und Kilo durchschnittlich 0,019 g Kreatinin, die den Körper mit dem Harn verlassen.

1) Amer. journ. of physiol. Vol. XIII. p. 45.

2) Amer. journ. of physiol. Vol. XIV. p. 252. 
van Hoogenhuyze und Verploegh ${ }^{1}$ ), die ihre Untersuchungen an sich selbst ausführten, bestimmten bei kreatininfreier Kost die pro Tag und Kilo mit dem Harn ausgeschiedene Kreatininmenge für den Ersteren za 0,020, für den Letzteren zu $0,027 \mathrm{~g}$

Wenn wir nun das Mittel aus allen angeführten Untersuchungen und den meinigen erheben, so finden wir, dass ein gesunder Mann in 24 Stunden durchschnittlich $0,021 \mathrm{~g}$ Kreatinin pro Kilo Körpergewicht mit dem Harn verliert, die endogenen Ursprungs sind.

Erheblich weniger Kreatinin wird vom weiblichen Geschlechte mit dem Harn zur Ausscheidung gebracht und zwar, wie schon erwähnt, sowohl absolut, als auch relativ.

Ich selbst verfüge nur über drei Fälle, in denen ich bei Franen die Kreatininausscheidung bei kreatininfreier Nahrung bestimmen konnte. Aus diesen Bestimmungen ergab sich, dass von ihnen in 24 Stunden bei der angegebenen Kost durchschnittlich nur $0,68 \mathrm{~g}$ Kreatinin den Körper mit dem Harn verliessen, was pro Tag und Kilo Körpergewicht nur 0,013 $\mathrm{g}$ ausmachte. Diese letztere Menge fanden auch Benedict und Myers ${ }^{2}$ ) im Mittel aus 26 von ihnen beobachteten Fällen. Folin (l. c.) dagegen bestimmte bei Frauen im Mittel aus 7 Fällen $0,009 \mathrm{~g}$ pro Tag und Kilo.

Nehmen wir auch hier das Mittel aus allen angeführten Untersuchungen, so kommen wir zu dem Ergebnis, dass von Frauen in 24 Stunden pro Kilo nur $0,012 \mathrm{~g}$, also nur etwa balb so viel Kreatinin mit dem Harn ausgeschieden wird, als von Männern.

Zum Vergleich mit den von mir gefundenen Zahlen habe ich nur die Resultate derjenigen Bestimmungen herangezogen, die nach der Folin'schen Methode, derer auch ich mich bediente, ausgeführt sind.

Die älteren Untersuchungen, bei denen das Neubauer'sche bzw. das Neubauer-Salkowski'sche Verfahren zur Kreatininbestimmung in Anwendung kam, habe ich nicht berücksichtigt, da, wie nunmehr durch die Untersuchungen von van Hoogenhuyze und Verploegh (l. c.), af Klercker ${ }^{3}$ ) u. a. sichergestellt ist, diese Methoden der gehörigen Genauigkeit entbehren, wodurch sich wohl die widersprechenden Angaben älterer Forscher über die Kreatininausscheidung beim Menschen erklären lassen.

1) Zeitschr. f. physiolog. Chemie. Bd. 46. S. 415.

2) Amer. journ. of physiol. 1907. Vol. XVIII.

3) Biochem. Zeitschr. Bd. 3. S. 45 . 
Was das Kreatin anlangt, so war bis in die neueste Zeit die allgemein herrschende Anschauung die, dass unter normalen, physiologischen Bedingungen diese Verbindung im Harne nicht erscheint, auch nicht bei Fleischnahrung, wofern nicht Fleisch oder Fleischbrühe, und mit ihnen natürlich neben dem Kreatinin auch Kreatin, in unmässigen Mengen aufgenommen werden.

Dieser Satz ist bezüglich des Harnes von Männern bis heute nicht beanstandet worden. Unter pathologischen Bedingungen (Diabetes mellitus, Fieber) jedoch tritt das Kreatin nicht selten im Harne auf, wie namentlich durch Untersuchungen aus neuerer Zeit erwiesen ist.

Bezüglich des Harnes von Frauen behaupten Krause und Cramer ${ }^{1}$ ), dass derselbe auch unter physiologischen Bedingungen bei kreatin- und kreatininfreier Nahrung Kreatin enthält und zwar am meisten gleich nach der Menstruation. Alsdann sinkt die Kreatinausscheidung allmählich, um in einigen Fällen, aber nicht in allen, bis auf 0 zurückzugehen. Sie sagen wörtlich Folgendes:

"Creatine is always present in the urine of women (living on a creatinine and creatine free diet) at one period of the sexual cycle, viz. immediatly after menstruation, and therefore presumably also during menstruation. In the intervening period it diminishes and may completely disappear in some individuals, while in others it persists."

Zur Illustration führen sie in einer Tabelle die Untersuchungsresultate eines Falles an, den sie als "the most typical case" bezeichnen.

Dieser Tabelle entnehme ich die uns interessierenden Daten, die ich in Folgendem wiedergebe. Neben die Kreatinzahlen stelle ich in Klammern die entsprechenden Werte als Kreatinin ausgedrückt.

Es handelt sich um eine unverheiratete Frau von 40 Jahren mit einem Körpergewicht von 59,7 Kilo, die auf kreatin- und kreatininfreie Nahrung gesetzt war. Der Harn wurde einmal vor und an fünf Tagen nach der Menstruation untersncht. Das Ergebnis war folgendes:

$\begin{array}{ccccc}\text { Datum } & \begin{array}{c}\text { Harnmenge } \\ \text { in cem }\end{array} & \begin{array}{c}\text { Kreatioin } \\ \text { in g }\end{array} & \begin{array}{c}\text { Kreatin } \\ \text { in g }\end{array} & \begin{array}{c}\text { Kreatin als } \\ \text { Kreatinin in g }\end{array} \\ \text { 12. I. 11 } & \begin{array}{c}1275 \\ \text { Menses vom }\end{array} & 0,905 & 0,0 & 0,0 \\ \text { 21. -23. I. } & & & \\ \text { 26. I. 11 } & 1510 & 0,995 & 0,042 & 0,036 \\ \text { 2. I. 11 } 11 & 1200 & 0,977 & 0,053 & 0,046 \\ \text { 10. II. } 11 & 1500 & 0,901 & 0,044 & 0,038 \\ & 1600 & 0,768 & 0,040 & 0,034\end{array}$

1) Journ. of physiol. Vol. XLII. Proc. oft the physiol soc. p. 34 . 
Krause und Cramer geben in ihrer Mitteilung nicht an, wie sie das Kreatinin und Kreatin bestimmten, ich darf aber wohl annehmen, dass sie die jetzt übliche, von Folin'1) vorgeschlagene, Methode benutzten. Von dieser Annahme ausgehend werde ich später noch einmal auf die angeführten Daten zurückkommen und sie einer eingehenderen Besprechung unterziehen.

Zunächst möchte ich aber noch einige andere nicht uninteressante Beobachtungen dieser Forscher erwähnen.

In ihrer Mitteilung "The occurrence of creatin in diabetic urine" ${ }^{2}$ ) sagen sie unter Anderem "Creatin was also found to be present in all the cases of pregnancy which were examined (8 cases). All these cases were in the seventh or eight month of pregnancy and did not present any abnormalities." Irgendwelche Zahlenangaben werden nicht gemacht.

In der späteren Mitteilung, der auch die obenangeführten Zahlenangaben entnommen sind und die den Titel "Sex and metabolism" trägt (l. c.), verweisen sie auf weitere fünf Fälle, in denen sie den Harn normaler schwangerer Frauen untersuchten und zu dem gleichen Ergebnis gelangten. In allen 13 Fällen wurde somit von den schwangeren Frauen in der angegebenen Periode der Schwangerschaft (7.-8. Monat) mit dem Harn neben Kreatinin auch Kreatin ausgeschieden. Die tägliche Kreatinausscheidung schwankte im Allgemeinen zwischen 0,05 und $0,1 \mathrm{~g}$. Als Maximum sind $0,15 \mathrm{~g}$ notiert.

Weiter führen Krause und Cramer an, dass in einem von ihnen untersuchten Falle in der fünften Woche post partum die Kreatinausscheidung mit dem Harn wohl vermindert, nicht aber aufgehoben erschien und selbst drei Monate nach der Geburt liess sich im Harn noch Kreatin nachweisen.

Die Befunde von Krause und Cramer an Schwangeren werden von van Hoogenhuyze und ten Doeschate ${ }^{3}$ ) bestätigt.

van Hoogenhuyze und ten Doeschate führten eine vielseitige Analyse des Harnes von Schwangeren aus; ich werde mich jedoch nur auf die Wiedergabe ihrer Kreatin- und Kreatininbestimmungen im Harn gesunder schwangerer Frauen beschränken. Im Ganzen liegen 6 derartige Fälle vor und zwar je einer aus

1) Zeitschr. f. physiol. Chemie. Bd. 41. S. 223.

2) Journ. of physiol. Vol. XL. Proc. of the physiol. soc. p. 51.

3) Ann. de gynéoologie et d'obstétrique. 1911. T. Vill. 
478 Wakulenko, Kreatinin- und Kreatinausscheidung durch den Harn.

dem 4., 8., und 9. Monat und drei aus dem 10. Monat. In allen diesen Fällen wies der Harn neben dem Kreatinin zweifelsohne auch Kreatin auf. In 3 von diesen Fällen waren die Bestimmungen auch auf die Wöchnerinnen ausgedehnt.

Es sei mir gestattet, in Kürze die für uns wichtigen Daten in Folgendem zusammenzustellen. Zur Erläuterung und um Missverständnissen vorzubeugen, sei gesagt, dass ich die von den genannten Forschern als Kreatinin angeführten Kreatinzahlen auf Kreatin umgerechnet habe. Die römischen Zahlen geben die Tabellen von van Hoogenhuyze und ten Doeschate an.

Fall XV. Schwangerschaft im 4. Monat.

\begin{tabular}{|c|c|c|c|c|}
\hline Datum & $\begin{array}{l}\text { 24-stündige } \\
\text { Harnmenge }\end{array}$ & $\begin{array}{l}\text { Gesamt- } \\
\text { kreatinin }\end{array}$ & $\begin{array}{c}\text { Präformiertes } \\
\text { Kreatinin }\end{array}$ & Kreatin \\
\hline 7. XII. & 1535 & 1,072 & 1,002 & 0,081 \\
\hline 8. XII. & 2050 & 0,965 & 0,874 & 0,106 \\
\hline 9. XII. & 1940 & 1,166 & 0,982 & 0,213 \\
\hline 10. XII. & 1515 & 1,057 & 0,990 & 0,078 \\
\hline 11. XII. & 1350 & 1,072 & 0,959 & 0,131 \\
\hline 12. XII. & 1360 & 0,744 & 0,715 & 0,037 \\
\hline 13. XII. & 1705 & 1,063 & 0,920 & 0,166 \\
\hline \multirow[t]{2}{*}{ 15. XII. } & 2190 & 1,206 & 1,123 & 0,094 \\
\hline & Mittel 1705 & 1,043 & 0,946 & 0,113 \\
\hline \multicolumn{5}{|c|}{ 31. V. Geburt des Kindes. } \\
\hline 8. VI. & 1450 & 1,678 & 1,450 & 0,265 \\
\hline 10. VI. & 1600 & 1,301 & 1,098 & 0.235 \\
\hline 11. VI. & 1300 & 1,088 & 0,986 & 0,118 \\
\hline 12. VI. & 2300 & 1,415 & 1,331 & 0,144 \\
\hline 13. VI. & 1800 & 1,105 & 1,012 & 0,108 \\
\hline 14. VI. & 1850 & 1,932 & 1,919 & 0,015 \\
\hline
\end{tabular}

Fall XVI. Schwangerschaft im 8. Monat

\begin{tabular}{|c|c|c|c|c|}
\hline 9. III. & 1250 & 1,226 & 1,070 & 0,181 \\
\hline 10. III. & 1500 & 1,074 & 0,935 & 0,161 \\
\hline 11. III. & 1275 & 0,890 & 0,783 & 0,124 \\
\hline 12. III. & 1165 & 1,110 & 1,015 & 0,110 \\
\hline 13. III. & 1765 & 0,893 & 0,835 & 0,067 \\
\hline \multirow[t]{2}{*}{ 14. III. } & 1585 & 1,019 & 0,917 & 0,118 \\
\hline & Mittel 1423 & 1,034 & 0,926 & 0,127 \\
\hline $\begin{array}{l}\text { 9. V. } \\
\text { 17. V. } \\
\text { 18. V. }\end{array}$ & $\begin{array}{c}\text { Geburt des Kindes. } \\
2200 \\
1250\end{array}$ & $\begin{array}{l}1,650 \\
1,332\end{array}$ & $\begin{array}{l}1,599 \\
1,272\end{array}$ & $\begin{array}{l}0,059 \\
0,070\end{array}$ \\
\hline
\end{tabular}

Fall XVII. Schwangerschaft im 9. Monat.

\begin{tabular}{|c|c|c|c|c|}
\hline 9. III. & 2050 & 1,065 & 0,938 & 0,147 \\
\hline 10. III. & 1520 & 0,844 & 0,692 & 0,176 \\
\hline 11. III. & 2370 & 1,116 & 0,994 & 0,258 \\
\hline 12. III. & 2360 & 0,956 & 0,839 & 0,135 \\
\hline 13. III. & 2030 & 1,054 & 0,904 & 0,174 \\
\hline \multirow[t]{2}{*}{ 14. III. } & 2150 & 1,062 & 0,880 & 0,211 \\
\hline & 2080 & 1,016 & 0,875 & 0,184 \\
\hline
\end{tabular}


Wakulenko, Kreatinin- und Kreatinausscheidung durch den Harn.

Fall XVIII. Schwangerschaft im 10. Monat.

$\begin{array}{lcccc}\text { Datum } & \begin{array}{c}\text { 24-stündige } \\ \text { Harnmenge }\end{array} & \begin{array}{c}\text { Gesamt- } \\ \text { kreatinin }\end{array} & \begin{array}{c}\text { Präformiertes } \\ \text { Kreatinin }\end{array} & \text { Kreatin } \\ \text { 22. II. } & 1780 & 1,502 & 1,360 & 0,165 \\ \text { 23. II. } & 1950 & 1,426 & 1,286 & 0,174 \\ \text { 24. II. } & 1940 & 1,786 & 1,641 & 0,168 \\ \text { 25. II. } & 1980 & 1,765 & 1,573 & 0,223 \\ \text { 26. II. } & 2089 & 1,755 & 1,504 & 0,291 \\ \text { 27. II. } & 2040 & 1,434 & 1,204 & 0,267 \\ & \text { Mitel } 1980 & 1,613 & 1,428 & 0,215\end{array}$

Fall XIX. Schwangerschaft im 10. Monat.

\begin{tabular}{rrrrr} 
7. V. & 2220 & 1,274 & 1,221 & 0,062 \\
8. V. & 2250 & 1,190 & 1,134 & 0,065 \\
9. V. & 2120 & 1,165 & 1,060 & 0,122 \\
10. V. & 2580 & 1,372 & 1,244 & 0,148 \\
\cline { 2 - 5 } & Mittel 2320 & 1,250 & 1,165 & 0,099 \\
17. V. & Geburt des Kindes. & & & \\
25. V. & 1450 & 1,250 & 1,149 & 0,117 \\
26. V. & 1500 & 0,892 & 0,802 & 0,104 \\
27. V. & 1130 & 0,762 & 0,693 & 0,080 \\
28. V. & 2250 & 1,470 & 1,375 & 0,110 \\
29. V. & 1450 & 1,172 & 1,108 & 0,074 \\
30. V. & 1970 & 1,500 & 1,494 & 0,007
\end{tabular}

Fall XX. Schwangerschaft im 10. Monat.

$\begin{array}{rcccc}\text { 28. V. } & 1860 & 1,506 & 1,325 & 0,314 \\ \text { 29. V. } & 1750 & 1,417 & 1,312 & 0,122 \\ \text { 30. V. } & 2400 & 1,389 & 1,279 & 0,128 \\ \text { 31. V. } & 1900 & 1,492 & 1,374 & 0,137 \\ \text { 1. VI. } & 1200 & 0,897 & 0,831 & 0,077 \\ & \text { Mittel } 1855 & 1,340 & 1,024 & 0,156 \\ \text { 2. VI. } & \text { Geburt des Kindes. } & & & \\ \text { 8. VI. } & 1000 & 0,792 & 0,648 & 0,167 \\ \text { 10. VI. } & 1200 & 0,933 & 0,823 & 0,128 \\ \text { 11. VI. } & 1600 & 1,349 & 1,306 & 0,050 \\ \text { 12. VI. } & 1430 & 1,192 & 1,181 & 0,013\end{array}$

Diese sehr wertvollen Untersuchungen lassen keinen Zweifel darüber, dass während der Gravidität mit dem Harn stets Kreatin neben Kreatinin ausgeschieden wird. Doch schwanken die von van Hoogenhuyze und ten Doeschate angegebenen Werte innerhalb sehr weiter Grenzen.

Wenn wir nur die 3 Fälle aus der gleichen Zeit der Schwangerschaft (10. Monat) mit einander vergleichen, so sehen wir, dass in dem einen Falle im Mittel pro Tag 0,215 $\mathrm{g}$ Kreatin im Harn erschienen, im zweiten Falle $0,099 \mathrm{~g}$ und im dritten $0,156 \mathrm{~g}$. Es ist sehr zu bedauern, dass in keinem von den angeführten Fällen das Körpergewicht der Versuchspersonen notiert ist, weshalb der Leser kein genügendes Bild über die Intensität der Kreatinausscheidung erhält. 
Das Gesagte gilt natürlich auch für die Kreatininzahlen und in einigen Fällen scheint es ganz unmöglich mit mehr weniger grosser Sicherheit sich darüber auszusprechen, ob die Kreatininausscheidung im Vergleich zur Kreatininausscheidung ausserhalb der Gravidität vermehrt ist oder nicht. So z. B. im Falle XVII, wo im Mittel pro Tag nur $0,875 \mathrm{~g}$ Kreatinin gefunden wurden. Im Allgemeinen jedoch wird der Eindruck gewonnen, dass die Schwangerschaft zu einer vermehrten Ausscheidung des Kreatinins Anlass gibt.

Dieses steht nicht im Einklang mit den Beobachtungen von Népean Londridge, dessen Arbeit ich übrigens nur nach einem Referat im "Journal der Geburtshilfe und Frauenkrankheiten" (November 1908. Russisch) kenne. Hier heisst es, der Autor hoffte eine Vermehrung der Ausscheidung endogenen Kreatinins infolge der Involution des Uterus zu finden, was sich jedoch nicht bestätigte.

Die Wöchnerinnen wurden von van Hoogenhuyze und ten Doeschate nicht gleich in den ersten Tagen nach der Geburt zur Untersuchung herangezogen, sondern erst nach 6 bis 8 Tagen.

Es darf aus ihren Analysen wohl der Schluss gezogen werden, dass auch nach erfolgter Geburt die Kreatinausscheidung fortdauert, eine Tendenz zur Abnahme zeigend, so dass sie 10 bis 14 Tage nach der Geburt schon sehr gering ist und man annehmen kann, dass in der 3.-4. Woche schon kein Kreatin mehr ansgeschieden wird. Auch die Kreatininausscheidung ist verhältnismässig hoch.

Ueber die Ausscheidung von Kreatin bei Wöchnerinnen in den ersten Tagen nach der Geburt liegen, meines Wissens, Untersuchungen nicht vor und was die Ausscheidung des Kreatinins betrifft, so sind mir auch nur die oben erwähnten Angaben von Népean Londridge zu Gesicht gekommen, nach denen eine vermehrte Kreatinausscheidung nicht statthaben soll. Untersucht wurden von ihm die Wöchnerinnen in den ersten 9 Tagen nach der Geburt.

Daher wollte es mir wohl wert erscheinen, der Lösung dieser Frage durch eine Reihe von Untersuchungen näher zu treten, deren Resultate ich mir nunmehr vorzulegen erlaube.

Meine Untersuchungen führte ich an 15 Wöchnerinnen aus, die mir dank der Liebenswürdigkeit des Herrn Direktors der hiesigen geburtshilflich-gynäkologischen Universitätsklinik Prof. Dr. I. Grammatikati zur Verfügung standen. Die Versuchspersonen 
waren auf kreatinin- und kreatinfreie Diät gesetzt; die Nahrung bestand aus Milch, Brot, Butter, Kompot, Mannagrütze, Tee und Zucker. Der Harn wurde, um Beimengungen von Blut und Lochialsekret zu vermeiden, nicht spontan, sondern mittelst Katheters entleert und in 24stündigen Mengen - von $8 \mathrm{Uhr}$ morgens bis $8 \mathrm{Uhr}$ morgens - gesammelt. Die Geburten wie das Wochenbett verliefen normal.

Die Kreatinin- und Kreatinbestimmungen wurden von mir nach der Folin'schen kolorimetrischen Methode ausgeführt.

Zur Bestimmung des Kreatinins wurden genau $10 \mathrm{ccm} \mathrm{Harn}$ in einen Messkolben von 250 bzw. $500 \mathrm{ccm}$ Inhalt gebracht, $15 \mathrm{ccm}$ 1,2 proz. Pikrinsäurelösung und darauf $5 \mathrm{ccm} 10$ proz. Natriumhydratlösung hinzugefügt. Das Gemisch wurde umgeschüttelt und 5 Minuten stehen gelassen, in welcher Zeit die Rotfärbung ihre grösste Intensität erreicht. Alsdann wird Wasser, je nach der Konzentration des Harnes, bis auf 250 bzw. $500 \mathrm{ccm}$ aufgefüllt und sofort zur kolorimetrischen Bestimmung geschritten. Zu derselben benutzte ich das Kolorimeter von Dubosq.

Als Vergleichslösung dient halbnormale Kaliumbichromatlösung. Folin hat festgestellt, dass dieselbe bei einer Schichtdicke von $8 \mathrm{~mm}$ in ihrer Färbung und Intensität der Schichtdicke von $8,1 \mathrm{~mm}$ eines in beschriebener Weise hergestellten Kreatinin- bzw. HarnPikrinsäure-Natronlaugegemisches, das in $500 \mathrm{ccm} 10 \mathrm{mg}$ Kreatinin enthält, entspricht.

In den einen Zylinder des Kolorimeters wird die Kaliumbichromatlösung getan und auf eine Schichtdicke von $8 \mathrm{~mm}$ eingestellt und für die Dauer der Bestimmung unverändert in dieser Stellung belassen. In den anderen Zylinder kommt das zu untersuchende Gemisch. Nun wird die Schichtdické des Gemisches bestimmt, welche zur Erlangung vollkommener Farbengleichheit beider Hälften des Gesichtsfeldes des Kolorimeters notwendig ist. Wenn das Gemisch in $500 \mathrm{ccm} 10 \mathrm{mg}$ Kreatinin enthält, so ist, wie schon gesagt, zur Erzielung der Farbengleichheit eine Schichtdicke von $8,1 \mathrm{~mm}$ erforderlich. Folglich muss sich aus der gefundenen Schichtdicke des Gemisches die in $500 \mathrm{ccm}$ derselben enthaltene Kreatininmenge nach der Formel $\frac{8,1}{\mathrm{~s}} \cdot 10$ berechnen lassen. In dieser Formel bezeichnet $\mathrm{s}$ die gefundene Schichtdicke des Gemisches. Waren also zur Bestimmung $10 \mathrm{ccm}$ Harn genommen, so findet man somit in dieser Weise direkt die in ihnen 
enthaltene Kreatininmenge, ausgedrückt in Milligramm, falls das Gemisch auf $500 \mathrm{ccm}$ aufgefüllt war. Enthielt der Harn nur wenig Kreatinin und musste daher das Gemisch statt auf 500 nur bis auf 250 aufgefüllt werden, so hat man natürlich den gefundenen Kreatininwert durch 2 zu dividieren, umgekehrt, wenn der Harn konzentriert war und das Gemisch auf 1000 gebracht werden musste - mit 2 zu multiplizieren, um die in $10 \mathrm{ccm}$ Harn enthaltene Kreatininmenge zu finden.

Es darf nicht unerwähnt gelassen werden, dass genaue Resultate nur dann erzielt werden, wenn die Beobachtungen innerhalb gewisser Grenzen der Schichtdicke des Gemisches sich bewegen. Je grösser die Abweichung von der Schichtdicke von 8,1 $\mathrm{mm}$ nach oben oder nach unten geht, um so ungenauer werden die Resultate. Daher rät Folin, die Bestimmungen in einer Schichtdicke auszuführen, die zwischen 5 und $13 \mathrm{~mm}$ liegt. Ich würde 5 und $11 \mathrm{~mm}$ als die Grenzwerte bezeichnen.

Die Folin'sche Methode dient nicht nur zur Bestimmung des Kreatinins, sondern kann auch zur Bestimmung des Kreatins benutzt werden. Das Kreatin muss aber zunächst in Kreatinin übergeführt werden. $\mathrm{Zu}$ diesem Zwecke empfiehlt Folin $10 \mathrm{~cm}$ Harn mit $20 \mathrm{ccm} \mathrm{N-Salzsäure} \mathrm{zu} \mathrm{versetzen} \mathrm{und} \mathrm{das} \mathrm{Gemisch} 21 / 2$ bis 3 Stunden auf dem Dampfbade zu digerieren. Nach der angegebenen Zeit wird das vorher abgekühlte Gemisch mit N-Natronlauge genau neutralisiert und im Uebrigen genau so verfahren, wie bei der Kreatininbestimmung.

Auf den Rat des Herrn Prof. Dr. F. Krüger, Leiter des hiesigen medizinisch-chemischen Universitätslaboratoriums, wich ich von dieser Vorschrift insofern ab, als ich zu $10 \mathrm{ccm}$ Harn nicht $20 \mathrm{ccm}$ N-Salzsäure, sondern $5 \mathrm{ccm}$ einer gegen die 10 proz. Natronlauge titrierten Salzsäurelösung fügte, und nicht $21 / 2$ bis 3 , sondern nur $1 \frac{1}{2}$ Stunden im Dampfbade digerierte und das Gemisch mit $5 \mathrm{cem}$ der 10 proz. Natronlauge neutralisierte. Die daraulfolgende Kreatininbestimmung wurde ganz nach den Vorschriften Folin's ausgeführt. Es werden also im Grunde genommen für jeden Harn zwei Kreatininbestimmungen gemacht - die eine vor, die andere nach der Behandlung des Harnes mit Salzsäure.

Aus der Differenz dieser beiden Bestimmungen ergibt sich die Kreatininmenge, die dem mit dem Harn ausgeschiedenen Kreatin entspricht

Um aus der gefundenen Kreatininmenge die Quantität des 
Kreatins zu berechnen, hat man eine Multiplikation mit 1,16 auszuführen.

Durch das Digerieren des Harnes. mit Salzsäure werden bekanntlich die Harnpigmente derart verändert, dass der Harn eine dunkelbraune Farbe annimmt. Diese Verfärbung des Harnes beeinflusst die Genauigkeit der kolorimetrischen Bestimmung nicht wesentlich, doch würde man, meiner Ansicht nach, fehlgehen, wenn man behaupten wollte, dass sie ganz ohne Einfluss sei.

Die beschriebene Folin'sche Methode der Kreatininbestimmung hat, wie allgemein anerkannt, gegenüber der Chlorzinkkreatininmethode nicht nur den Vorteil der Einfachheit und Schnelligkeit der Ausführung, sondern zugleich auch den weit grösserer Genauigkeit. Selbstredend stellen sich aber ihrer Genauigkeit gewisse Grenzen, denn es gibt keine absolut genauen analytischen Methoden, innerhalb gewisser Fehlergrenzen bewegen sich alle Bestimmungen. Das darf auch hier nicht ausser Acht gelassen werden, umso weniger, als bei der Umrechnung auf die Tagesmengen man Multiplikationen mit grossen Zahlen auszuführen hat. Ganz besonders muss das in den Fällen berücksichtigt werden, wo neben den Kreatinin- auch Kreatinbestimmungen auszuführen sind. Man könnte sonst leicht auf Kreatingehalt schliessen, wo dasselbe nicht vorhanden ist.

Es ist bekannt, dass die bei der kolorimetrisehen Bestimmung benutzte Lichtquelle eine grosse Bedeutung hat. Bei diesen Bestimmungen ist es angeraten, diffuses Tageslicht anzuwenden.

Es ist ferner bekannt, dass bei der Kreatininbestimmung nach Folin die Konzentration der zu untersuchenden Kreatininlösung die Genauigkeit der Bestimmung beeinflusst. Daher wird verlangt, dass bei der üblichen Einstellung der Vergleichslösung die zur Erzielung der Farbengleichheit nötige Schichtendicke der Kreatininlösung nicht zu weit vom Normalpunkt $(8 \mathrm{~mm})$ abweicht. Sie soll im Allgemeinen nicht über 11 und nicht unter $5 \mathrm{~mm}$ gehen. Je grösser die Abweichung vom Normalpunkt ist, umso ungenauer fällt die Bestimmung aus.

Es ist endlich bekannt, dass die Jaffé'sche Reaktion, und mit dieser haben wir es doch bei der Folin'schen Methode zu tun, je nach der Temperatur, bei welcher sie ausgeführt wird, eine verschiedene Intensität der für das Kreatinin charakteristischen Rotfärbung, bei gleichem Gehalt der Lösung an Kreatinin, zeigt: je höher die Temperatur, umso intensiver die Färbung. Daher 
muss die Reaktion stets bei annähernd derselben Temperatur (etwa $20^{\circ}$ C.) aller Reagentien vorgenommen werden.

Diesen letzteren Umstand möchte ich mit Bezug auf die Kreatinbestimmungen ganz besonders unterstreichen. Es wird ja verlangt, dass das Harn-Salzsäuregemisch, nachdem es dem Dampfbade entnommen, abgekühlt wird, bevor man an den Zusatz der Reagentien geht. Diese Vorschrift wird ohne Zweifel immer befolgt. Es darf aber nicht unbeachtet bleiben, - und darauf wird vielleicht zu wenig Rücksicht genommen, - dass beim Neutralisieren des abgekühlten Harn-Salzsäuregemisches wiederum eine nicht unbeträchtliche Menge Wärme frei wird. Man muss daher, will man möglichst genaue Resultate erzielen, mit dem weiteren Zusatz der Reagentien warten, bis die erforderliche Temperatur sich eingestellt hat, mit anderen Worten, zum zweiten Male abkühlen.

Man sieht aus dem Gesagten, dass die Genauigkeit der kolorimetrischen Bestimmung von einer Reihe von Momenten beeinflusst werden kann. Das setzt den grossen Wert der Folin'schen Methode natürlich nicht herab, da bei sorgfältiger Arbeit alle diese Fehlerquellen sich leicht bis auf ein Minimum reduzieren lassen. Sie dürfen aber nie aus dem Auge gelassen werden und deswegen habe ich sie etwas eingehender besprochen.

Abgesehen von den genannten Fehlerquellen, die nach Möglichkeit zu umgehen sind, und ganz unabhängig von ihnen bewegt sich die kolorimetrisehe Bestimmung als solche innerhalb gewisser Fehlergrenzen, die bei den Kreatinbestimmungen nicht unberücksichtigt bleiben dürfen.

Folin verlangt, dass in einer Bestimmungsreihe die Einzelbestimmungen der Schichtendicke um nicht mehr als $0,3 \mathrm{~mm}$ von einander und $0,1 \mathrm{~mm}$ vom Mittel aus 5 bis 10 Einzelbestimmungen abweichen sollen. Bei einiger Uebung lässt sich dieses sehr wohl erreichen.

Der wahrscheinliche Fehler für die kolorimetrischen Kreatininbestimmungen im Harn nach Folin ist, soviel ich weiss, nicht bestimmt worden. Auch ich habe besondere Versuche nach dieser Seite nicht angestellt, doch glaube ich auf Grund meiner Erfahrungen nicht fehlzugreifen, wenn ich annehme, dass er, in Anbetracht unvermeidlicher kleiner Fehler bei der Abmessung des Harnes, beim Auffüllen des Messkolbens mit Wasser und dergleichen 
mehr, für das Mittel aus 5 bis 10 Einzelbestimmungen der Schichtdicke, etwa $0,1 \mathrm{~mm}$ ausmacht. Mit anderen Worten, man würde für einen Harn, der 0,1 pCt. Kreatinin enthält, in 2 verschiedenen Analysen, statt der verlangten Schichtdicke von $8,1 \mathrm{~mm}$, das eine Mal eine Schichtdicke von 8,0 und das andere Mal ron $8,2 \mathrm{~mm}$ finden können. Nehmen wir nun an, dass die 24 stündige Harnmenge $1500 \mathrm{ccm}$ betrug, so würde die ausgeschiedene Kreatininmenge nach der einen Bestimmung 1,519, nach der anderen 1,482 g ausmachen, was eine Differenz von 0,037 gibt.

Findet man nun eine solche Differenz bei dêr Untersuchung des Harnes vor und nach seiner Digestion mit Salzsäure, so wäre es entschieden gewagt, daraus den Schluss zu ziehen, dass der Harn neben Kreatinin Kreatin enthält.

Mit Sicherheit kann man, meiner Meinung nach, auf Anwesenheit von Kreatin im Harn nur dann schliessen, wenn sich bei der kolorimetrischen Untersuchung vor und nach der Behandlung mit Salzsäure eine Differenz von mehr als $0,3 \mathrm{~mm}$ Schichtdicke herausstellt. Im entgegengesetzten Falle muss die Frage nach der Gegenwart von Kreatin sub dubio belassen werden.

Nach diesen Ausführungen sei mir gestattet, noch einmal auf die oben zitierten Angaben von Krause und Cramer bezüglich des Auftretens von Kreatin im Harne von normalen Frauen zurückzukommen.

Wenn wir die Zahlen der oben wiedergegebenen Tabelle ansehen, so finden wir, dass die pro Tag ausgeschiedenen Kreatinmengen nur sehr gering sind - sie schwanken, in Kreatininwerten ausgedrückt, zwischen 0,034 und $0,046 \mathrm{~g}$.

Der Prozentgehalt der von ibnen untersuchten Harne an Kreatinin ist ein relativ geringer - er liegt zwischen 0,048 und 0,081 . Daher lässt sich annehmen, dass Krause und Cramer, um nicht an der äussersten und weniger sichere Resultate gebenden Grenze der Schichtendicke, die von Folin gestattet wird, zu arbeiten, die Harne vom 26. I. und rom 2. und 10. II. (bei Bearbeitung von $10 \mathrm{ccm}$ zur Kreatininbestimmung) nicht auf 500, sondern nur auf $250 \mathrm{ccm}$ brachten.

Von dieser Annahme ausgehend berechnete ich, welche Schichtendicken sie vor und nach der Behandlung der Harne bei ihren kolorimetrischen Bestimmungen gefunden haben müssen. Die Rechnung ergab folgendes: 
486 Wakulenko, Kreatinin- und Kreatinausscheidung durch den Harn.

$\begin{array}{rccc}\text { Datum } & \text { Präform. Kreatinin } & \text { Gesamtkreatin } & \text { Differenz } \\ \text { 26. I. } & 6,2 \mathrm{~mm} & 5,9 \mathrm{~mm} & 0,3 \mathrm{~mm} \\ \text { 27. I. } & 10,0 " & 9,5 \% & 0,5 \% \\ \text { 2. II. } & 6,7 " & 6,5 " & 0,2, \\ \text { 10. II. } & 8,4 " & 8,0 " & 0,4 ",\end{array}$

Wie ersichtlich, liegen die angeführten Differenzen in zwei Analysen innerhalb der Fehlergrenzen der Methode, während sie in den beiden anderen Analysen nur ganz unbeträchtlich über sie hinausgehen.

Und dabei ist dieser Fall, wie Krause und Cramer selbst sagen, ,the most typical case". Man ist also berechtigt anzunehmen, dass in ihren übrigen Fällen die Differenzen zwischen den kolorimetrischen Ablesungen vor und nach Behandlung des Harnes mit Salzsäure noch geringer sind, d. h. sich durchweg in den Grenzen der der Methode anhaftenden Fehler bewegen.

Ich will durchaus nicht die Möglichkeit in Abrede stellen, dass der Harn von Frauen während und in den ersten Tagen nach der Menstruation Kreatin enthält; ich halte es sogar für wahrscheinlich. Beweisend erscheinen mir jedoch die Ergebnisse von Krause und Cramer nicht, jedenfalls bedürfen ihre Angaben einer weiteren Bestätigung und sind zunächst mit Vorsicht aufzunehmen. Ganz besonders gilt dieses von der Ausscheidung des Kreatins mit dem Harn während der intermenstrualen Perioden. Warum sich auch in dieser Zeit der Stoffwechsel im Organismus des Weibes anders verhalten soll, als im Organismus des Mannes, ist mir nicht recht verständlich.

Ich gehe nun zur Wiedergabe meiner Untersuchungsergebnisse über.

Bei der Angabe der Reaktion des Harnes bedeutet $\mathrm{s}=$ sauer, ss $=$ schwach sauer, $a=$ alkalisch, sa $=$ schwach alkalisch und $\mathrm{n}=$ neutral.

Fall I. IV para, 27 Jahre. Geschlecht des Kindes männlich.

$\begin{array}{lcc}\text { Tag nach der Geburt } & 1 . & 2 . \\ 24 \text { stündige Harnmenge } & 1850 & 2470 \\ \text { Reaktion des Harnes } & \text { ss } & \text { ss } \\ \text { Spez. Gewicht des Harnes } & 1013 & 1012 \\ \text { Kreatinin in Gramm } & 1,43 & 1,82 \\ \text { Kreatin in Gramm } & 0,41 & 0,61\end{array}$

Fall II. II para, 45 Jahre. Geschlecht des Kindes männlich.

$\begin{array}{lcc}\text { Tag nach der Geburt } & 1 . & 2 . \\ \text { 24 stündige Harnmenge } & 1730 & 1980 \\ \text { Reaktion des Harnes } & \text { ss } & \text { ss } \\ \text { Spez. Gewicht des Harnes } & 1012 & 1010 \\ \text { Kreatinin in Gramm } & 1,06 & 1,05 \\ \text { Kreatin in Gramm } & 0,45 & 0,56\end{array}$


Fall III. VI para, 34 Jahre. Geschlecht des Kindes weiblich.

$\begin{array}{lcc}\text { Tag nach der Geburt } & 1 . & 3 . \\ \text { 24 stündige Harnmenge } & 2210 & 2230 \\ \text { Reaktion des Harnes } & \mathrm{sa} & \mathrm{sa} \\ \text { Spez. Gewicht des Harnes } & 1007 & 1011 \\ \text { Kreatinin in Gramm } & 0,92 & 0,91 \\ \text { Kreatin in Gramm } & 0,20 & 0,46\end{array}$

Fall IV. IX para, 30 Jahre. Geschlecht des Kindes männlich.

$\begin{array}{lcc}\text { Tag nach der Geburt } & 1 . & 2 . \\ \text { 24 stündige Harnmenge } & 3046 & 1170 \\ \text { Reaktion des Harnes } & \text { ss } & \text { ss } \\ \text { Spez. Gewicht des Harnes } & 1007 & 1018 \\ \text { Kreatinin in Gramm } & 0,73 & 0,74 \\ \text { Kreatin in Gramm } & 0,97 & 0,71\end{array}$

Fall V. IV para, 26. Jahre. Geschlecht des Kindes männlich.

$\begin{array}{lccc}\text { Tag nach der Geburt } & 1 . & 2 . & 3 . \\ \text { 24 stündige Harnmenge } & 1170 & 1540 & 650 \\ \text { Reaktion des Harnes } & \text { ss } & \text { ss } & \text { s } \\ \text { Spez. Gewicht des Harnes } & 1021 & 1013 & 1017 \\ \text { Kreatinin in Gramm } & 1,21 & 0,99 & 0,84 \\ \text { Kreatin in Gramm } & 0,57 & 0,48 & 0,26\end{array}$

Fall VI. II para, 19 Jahre. Geschlecht des Kindes weiblich.

Tag nach der Geburt 1.

24 stündige Harnmenge 2400

Reaktion des Harnes ss

Spez. Gewicht des Harnes 1010

Kreatinin in Gramm 1,38

Kreatin in Gramm $\quad 0,99$

In den bisher angeführten 6 Fällen war leider das Körpergewicht der Wöchnerinnen nicht bestimmt worden. Die folgenden Fälle haben diese Lücke nicht.

Fall VII. VI para, 35 Jahre. Körpergew. $70 \mathrm{~kg}$. Geschlecht des Kindes männlich.

Tag nach der Geburt

24 stündige Harnmenge

Reaktion des Harnes

Spez. Gewicht des Harnes 1017

Kreatinin in Gramm $\quad 0,89$

Kreatin in Gramm $\quad 0,18$
1.

60

s

18

Fall VIII. VI para, 37 Jahre. Körpergew. $68 \mathrm{~kg}$. Geschlecht des Kindes männlich.

Tag nach der Geburt 1

24 stündige Harnmenge 1600

Reaktion des Harnes ss

Spez. Gewicht des Harnes 1012

Kreatinin in Gramm $\quad 1,02$

Kreatin in Gramm

Fall IX. II para, 21 Jahre. Körpergew. $57,7 \mathrm{~kg}$. Geschlecht des Kindes weiblich.

Tag nach der Geburt

24 stündige Harnmenge

Reaktion des Harnes

Spez. Gewicht des Harnes

Kreatinin in Gramm

Kreatin in Gramm
1.

1960

$\mathrm{s}$

1012

1,30

0,85
2.

990

SS

1014

0,92

0,45
3.

0,26 
Fall X. IV para, 27 Jahre. Eörpergewicht $60 \mathrm{~kg}$. Geschlecht des Kindes männlich.

$\begin{array}{lccc}\text { Tag nach der Geburt } & 1 . & 2 . & 3 . \\ \text { 24 stïndige Harnmenge } & 2140 & 715 & 740 \\ \text { Reaktion des Harnes } & \mathrm{ss} & \mathrm{sz} & \mathrm{n} \\ \text { Spez. Gewicht des Harnes } & 1008 & 1022 & 1015 \\ \text { Kreatinin in Gramm } & 0,94 & 0,87 & 0,74 \\ \text { Kreatin in Gramm } & 0,31 & 0,49 & 0,37\end{array}$

Fall XI. I para, 19 Jahre. Körpergewicht $48,5 \mathrm{~kg}$. Geschlecht des Kindes männlich.

$\begin{array}{lccc}\text { Tag nach der Geburt } & 1 . & 2 . & 3 . \\ \text { 24 stündige Harnmenge } & 2800 & 1460 & 1640 \\ \text { Reaktion des Harnes } & \text { sa } & \text { ss } & \text { ss } \\ \text { Spez. Gewicht des. Kindes } & 1010 & 1012 & 1008 \\ \text { Kreatinin in Gramm } & 1,47 & 1,00 & 0,74 \\ \text { Kreatin in Gramm } & 0,15 & 0,45 & 0,30\end{array}$

Fall XII. Vpara, 27 Jahre. Körpergewicht $66 \mathrm{~kg}$. Geschlecht des Kindes weiblich.

Tag nach der Geburt

24 stündige Harnmenge

Reaktion des Harnes

215

1.5

2.

2160

3 ,

Spez. Gewicht des Harnes

SS

Kreatinin in Gramm

1011

ss

1170

Kreatin in Gramm

1,16

1012

ss

$1,25 \quad 1,39$

0,31

0,60

0,39

Fall XIII. X para, 35 Jahre. Körpergewicht $43,6 \mathrm{~kg}$. Geschlecht des Kindes weiblich.

Tag nach der Geburl

24 stündige Harnmenge

Reaktion des Harnes

Spez. Gewicht des Harnes

Kreatinin in Gramm

Kreatin in Gramm

$\begin{array}{cc}1 & 2 \\ 1960 & 1970 \\ \text { Ss } & n \\ 1014 & 1008 \\ 1,21 & 0,62 \\ 0,35 & 0,27\end{array}$

$\begin{array}{cc}3 . & 4 \\ 1400 & 1210 \\ \text { ss } & \text { ss } \\ 1014 & 1013 \\ 0,78 & 0,77 \\ 0,25 & 0,27\end{array}$

Fall XIV. Vpara, 25 Jahre. Körpergewicht 56,9 g. Geseblecht des Kindes weiblich.

Tag nach der Geburt

24 stündige Harnmenge

Reaktion des Harnes

Spez. Gewicht des Harnes

Kreatinin in Gramm

Kreatin in Gramm

$\begin{array}{cccc}1 . & 2 . & 3 . & 4 . \\ 1390 & 1400 & 650 & 1020 \\ \text { ss } & \mathrm{n} & \mathrm{ss} & \mathrm{ss} \\ 1016 & 1012 & 1020 & 1014 \\ 1.09 & 1,29 & 0,94 & 1,31 \\ 0,79 & 0,73 & 0,20 & 0,16\end{array}$

Fall XV. I para, 22 Jahre. Körpergewicht $48,2 \mathrm{~kg}$. Geschlecht des Kindes männlich.

Tag nach der Geburt

24 stündige Harnmenge

Reaktion des Harnes

1
2150
ss

Spez. Gewicht des Harnes

1010

Kreatinin in Gramm

1,18

Kreatin in Gramm

0,16

5 420

3. 840

4.

ss

1019

1016

385

1.08

1,02

1029

0,17

0,39

0,77

0,26

Schon ein ganz flüchtiges Ueberblicken der mitgeteilten Beobachtungsprotokolle belehrt uns darüber, dass Wöchnerinnen mit dem Harne neben dem Kreatinin stets und dazu recht 
Wakulenko, Kreatinin- und Kreatinausscheidung durch den Harn.

bedeutende Mengen von Kreatin ausscheiden, die aber innerhalb sehr weiter Grenzen schwanken, von 0,15 (Fall XI, erster Tag nach der Geburt) und bis zu $0,99 \mathrm{~g}$ (Fall VI, zweiter Tag nach der Geburt) pro die.

In nachstehender Tabelle habe ich das von mir gewonnene Material in übersichtlicher Form zusammenzustellen versucht. $\mathrm{Zu}$ derselben bemerke ich, dass das Körpergewicht in Kilogrammen, die tägliche Harnmenge in Kubikzentimetern und die täglichen Kreatinin- und Kreatinausscheidungen in Grammen angegeben sind. Ausserdem ist in der Tabelle die von mir berechnete mittlere Tagesausscheidung der genannten Verbindungen pro Kilogramm angeführt.

\begin{tabular}{|c|c|c|c|c|c|c|c|c|c|c|c|c|c|}
\hline \multirow{3}{*}{$\underset{. \vec{\sigma}}{\vec{\sigma}}$} & \multirow{3}{*}{ 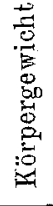 } & \multicolumn{12}{|c|}{ Tage nach der Geburt. } \\
\hline & & \multicolumn{3}{|c|}{1.} & \multicolumn{3}{|c|}{2.} & \multicolumn{3}{|c|}{3.} & \multicolumn{3}{|c|}{4.} \\
\hline & & $\underset{ت}{g}$ & 总. & 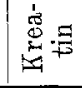 & 营 & 量 & 离. & 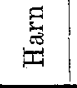 & 㿝 & 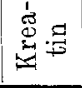 & 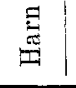 & 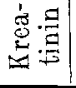 & 昰寻 \\
\hline 1 & - & 1850 & 1,43 & 0,41 & 2470 & 1,82 & 0,61 & - & - & - & - & - & - \\
\hline 2 & - & & 1,06 & 0,45 & - & $-\infty$ & - & 1980 & 1,05 & 0,56 & $\cdots$ & 一 & - \\
\hline 3 & - & & 0,92 & 0,20 & 2230 & 0,91 & 0,46 & - & - & - & - & - & - \\
\hline 4 & - & & 0,73 & 0,97 & 1170 & 0,74 & 0,71 & - & - & - & - & - & - \\
\hline 5 & - & & 1,21 & 0,57 & 1540 & 0,99 & 0,48 & 650 & 0,84 & 0,26 & - & - & - \\
\hline 6 & 一 & 240 & 1,31 & 0,99 & & - & - & -1 & - & - & - & - & - \\
\hline \multicolumn{2}{|c|}{ Mittel } & 2055 & 1,11 & 0,60 & 1850 & 1,11 & 0,57 & 1315 & 0,95 & 0,41 & - & - & - \\
\hline 7 & 70,0 & 760 & 0,89 & 0,18 & - & - & - & 一 & 一 & 一 & - & - & - \\
\hline 8 & 68,0 & 160 & 1,02 & 0,49 & 900 & 0,92 & 0,45 & - & - & - & - & 一 & - \\
\hline 9 & 57,7 & & 1,30 & 0,85 & 930 & 1,34 & 0,24 & - & - & - & - & 一 & - \\
\hline 10 & 60,0 & & 0,94 & 0,31 & 715 & 0,87 & 0,49 & 740 & 0,78 & 0,30 & - & - & $\ldots$ \\
\hline 11 & 48,5 & & 1,47 & 0,15 & 1640 & 1,00 & 0,4 & 1460 & 0,74 & 0,37 & - & - & - \\
\hline 12 & 66,0 & & 1,16 & 0,3 & 21 & 1,25 & 0,6 & & 1,39 & 0,39 & & - & \\
\hline 13 & 43,6 & & 1,21 & 0,3 & 19 & 0.62 & 0,27 & 1440 & 0,78 & 0,25 & 1210 & 0.77 & 0,27 \\
\hline 14 & 56,9 & & 1,09 & 0,79 & 1400 & 1,29 & 0,73 & 650 & 0,94 & {$[0,20$} & 1020 & 1,31 & 0,16 \\
\hline 15 & 58,2 & & & 0,16 & & & 0,17 & & 1,02 & 0,39 & & 0,77 & 0,26 \\
\hline \multicolumn{2}{|c|}{ Mittel } & 1880 & 1,14 & 0,36 & 1267 & 1,04 & 0,43 & 1050 & 0,94 & 0,32 & 905 & 0,95 & 0,23 \\
\hline \multicolumn{2}{|c|}{ oro Kilo } & & 0,019 & 0,006 & & 0,018 & 10,007 & & 0,017 & 0,006 & & $\mid 0,018$ & 0,00 \\
\hline
\end{tabular}

An der Hand dieser Tabelle tritt das soeben Gesagte auf das Deutlichste hervor. In keinem Falle und an keinem unserer Beobachtungstage fehlte das Kreatin im Harne. und selbst die kleinste von mir bestimmte Tagesmenge repräsentiert noch eine ganz respektable Grösse $(0,15 \mathrm{~g})$, im Mittel aber aus allen 38 Beobachtungstagen finde ich die Tagesausscheidung des Kreatins zu 0,42 g. 
Ganz ausserordentlich gross sind die Schwankungen in der Quantität des pro Tag mit dem Harn zur Ausscheidung gelangenden Kreatins. Dabei ist es mir nicht möglich gewesen, irgend eine Gesetzmässigkeit festzustellen. Auch ein prägnanter individueller Zug, eine individuelle Eigentümlichkeit lässt sich nicht zur Erklärung heranziehen, denn es handelt sich nicht darum, dass das eine Individuum viel Kreatin ausscheidet und das andere wenig, sondern um grosse Schwankungen bei einer und derselben Person. Als Beispiel möge der schon erwähnte Fall XI gelten, der am ersten Tage nach der Geburt die kleinste von mir beobachtete Kreatinmenge gab, nämlich $0,15 \mathrm{~g}$. Am darauffolgenden Tage, d. h. am zweiten Tage des Wochenbettes, schied die Frau eine $3 \mathrm{mal}$ grössere Menge, nämlich $0,45 \mathrm{~g}$ Kreatin aus. Aehnliche Sprünge sehen wir in einer ganzen Reihe von Fällen.

Wenn wir uns die Tabellen von van Hoogenhuyze und ten Doeschate näher ansehen, so können wir uns davon überzeugen, dass derartige Sprünge in der Kreatinausscheidung auch während der Schwangerschaft beobachtet werden. So z. B. werden von ihnen in Tabelle XX an einem Tage 0,077 und an einem anderen Tage $0,314 \mathrm{~g}$ Kreatin angeführt.

Im Allgemeinen ist die Menge des Kreatins, die im Laufe des Tages den Körper mit dem Harn verlässt, in den ersten Tagen des Wochenbettes ganz bedeutend grösser, als während der letzten Monate der Schwangerschaft. Ich habe aus den Angaben von van Hoogenhuyze und ten Doeschate für den IX. und X. Monat der Gravidität das Mittel für die in 24 Stunden ausgeschiedene Kreatinmenge mit $0,17 \mathrm{~g}$ berechnet. In meinen Untersuchungen beträgt es 0,42 . Es wird also in den ersten Tagen des Wochenbettes pro Tag 2,5 mal mehr Kreatin durch die Nieren zur Ausscheidung gebracht, als in derselben Zeit in den letzten Monaten der Schwangerschaft den Körper verlässt.

Inwieweit an dieser Ausscheidung des Kreatins eine event. Aenderung in der Tätigkeit der Leberzellen und eine Abweichung von der normalen Funktion der Leber beteiligt ist, wie das van Hoogenhuyze und ten Doeschate annehmen, will jch hier nicht einer weiteren Erörterung unterziehen. Die kolossalen Schwankungen und die sprungweise Steigerung und Herabsetzung in der Aus- 
scheidung des Kreatins scheinen mir durch solche Annahme nicht genügend erklärt werden zu können.

Weit davon entfernt, eine gewisse Beziehung der Leber zu diesem Prozesse absprechen zu wollen, kann ich mich nicht des Gedankens erwehren, dass die Kreatinausscheidung in einem direkten Zusammenhang mit der Kontraktion und Involution des Uterus und mit der Menge und Ausscheidung der Lochien steht.

Meines Wissens ist das Lochialexkret nicht auf die Gegenwart und den Gehalt an Kreatin nnd Kreatinin untersucht. Es wäre aber denkbar, dass diese Verbindungen in ihm vorhanden wären. Dann könnte aber ihre Resorption von der lädierten Innenfläche des Uterus sehr leicht von statten gehen. Sie würden in relativ grossen Mengen in den Kreislauf gelangen und durch die Nieren zur Ausscheidung gebracht werden können, namentlich, wenn zudem noch die das Kreatin dehydratierende Wirkung der Leber herabgesetzt ist. Unter dieser Voraussetzung liessen sich die grossen Schwankungen in der Kreatinausscheidung leicht erklären; aus einem gut kontrahierten Uterus würde in der Zeiteinheit weniger zur Resorption gelangen, als aus einem schlaffen Uterus, ebenso müsste bei Stagnation des Lochialexkretes der Uebergang ins Blut schneller und leichter vor sich gehen, als bei leichtem and unbehindertem Abfluss.

Ich wende mich nun zum Kreatinin. Dasselbe findet sich regelmässig im Harn, sowohl in dem von Männern, als auch in dem von Weibern, in dem der Ersteren aber in grösseren Mengen.

Wie eingangs erwähnt war, wird von Frauen bei kreatin- und kreatininfreier Kost in 24 Stunden im Mittel das Kreatinin in einer Quantität von $0,68 \mathrm{~g}$ ausgeschieden. Auf ein Kilogramm Körpergewicht kommen $0,012 \mathrm{~g}$.

Sehen wir uns nun unsere Tabelle an, so finden wir, dass die von Wöchnerinnen in den ersten Tagen nach der Geburt mit dem Harn ausgeschiedene Tagesmenge des Kreatinins bedeutend grösser ist. Im Mittel aus allen meinen Untersuchungen, in denen das Körpergewicht der Frauen bekannt war $(9$ Fälle mit $26 \mathrm{Be}-$ obachtungstagen) ergeben sich pro Tag und Kilo $0,018 \mathrm{~g}$, also um etwa 50 pCt. mehr, als bei Franen ausserhalb der Gravidität und des Wochenbettes.

Kurz meine Resultate zusammenfassend, darf, ich behaupten, dass in den ersten Tagen des Wochenbettes: 
492 Wakulenko, Kreatinin- und Kreatinausscheidung durch den Harn.

1. neben dem Kreatinin stets reichliche Mengen von Kreatin im Harn erscheinen and

2. die Ausscheidung des Kreatinins gegen die Norm um etwa 50 pCt. pro Tag and Kilo vermehrt ist.

Zum Schluss erfülle ich eine angenehme Pflicht, indem ich meinen hochverehrten Lehrern, den Herren Professoren J. Grammatikati und F. Krüger an dieser Stelle meinen herzlichen Dank ausspreche für ihr jederzeit liebenswürdiges Entgegenkommen und für ihre Hilfe mit Rat und Tat. 